\title{
The incidence of mismatch repair gene defects in colorectal liver metastases
}

\author{
RAUL ALVARADO-BACHMANN $^{1}$, ADRIAN SMITH ${ }^{2}$, JUSTIN S. GUNDARA ${ }^{1}$, SAMUEL C.L. KUO ${ }^{1}$, \\ ANTHONY J. GILL ${ }^{3}$, JASWINDER S. SAMRA ${ }^{1}$ and THOMAS J. HUGH ${ }^{1}$ \\ ${ }^{1}$ Upper Gastrointestinal Surgical Unit; ${ }^{2}$ Department of Colorectal Surgery; ${ }^{3}$ Department of Anatomical Pathology, \\ University of Sydney, Royal North Shore Hospital, Sydney, New South Wales 2065, Australia
}

Received January 22, 2014; Accepted March 29, 2014

DOI: $10.3892 / \mathrm{mmr} .2014 .2257$

\begin{abstract}
Knowledge of the molecular biology of primary colorectal cancer (CRC) has improved in recent years, and one reason for this is the identification of microsatellite instability (MSI), which occurs in up to $15 \%$ of sporadic CRC. However, less is known regarding the processes involved in colorectal liver metastases (CRLM). Increasing numbers of patients with CRLM are suitable for curative resection, so the identification of molecular markers may improve patient selection. The aim of the present study was to characterise the incidence of MSI in resected CRLM. Fifty-one sequentially resected CRLM specimens were selected. Clinicopathologic data was collated and immunohistochemistry for MLH1 and MSH2 was performed on paraffin sections of the CRLM specimens. The association between abnormal staining and the clinicopathological data was examined. The median age of the subjects in the current study was 65 years, the average number of CRLM was 2 and the median overall survival time was 42.1 months post liver resection. None of the 50 resected specimens demonstrated abnormal staining for MLH1 or MSH2. Compared with the previously reported incidence of MSI in primary CRC, the low incidence of MSI in the current cohort of CRLM precludes its use as a marker for use in making clinical decisions regarding this condition.
\end{abstract}

\section{Introduction}

Colorectal cancer (CRC) is the second and fifth most common type of cancer in females and males, respectively, in Australia (1). Worldwide, there are over a million cases diagnosed each year with a disease-specific mortality rate of $\sim 33 \%$ in the developed world (2). The majority of mortalities occur from metastatic disease, and the most common site for

Correspondence to: Dr Thomas J. Hugh, Upper Gastrointestinal Unit, Level 8A, University of Sydney, Royal North Shore Hospital, Pacific Hwy, St. Leonards, Sydney, New South Wales 2065, Australia E-mail: thugh@med.usyd.edu.au

Key words: colorectal cancer, mismatch repair, liver metastases metastases is the liver, where metastases develop in up to $40 \%$ of patients with CRC (3).

While treatment options for hepatic metastatic disease have rapidly expanded, the only hope for long-term survival is surgical resection. The range of available indicators for identifying cases in which potentially curative resection is possible has expanded significantly since initial reports and generally, $30-50 \%$ of patients have operable disease. Following surgery, five-year survival rates of $25-50 \%$ have been reported $(4,5)$.

Predictors of clinical outcome following liver resection have been thoroughly investigated and include the following: Primary tumour grade; number and size of liver metastases and whether they are synchronous or metachronous; period of disease-free survival; presence of extrahepatic disease; and resection margin $(6,7)$. These parameters have significant limitations and are based on population data rather than being individualized for each patient. Recent attention has therefore focused on the relevance of molecular prognostic markers (8-10).

The molecular changes associated with CRC development have been well characterized compared with those of most other types of cancer, and two main pathways have been described. The adenoma-carcinoma sequence, as proposed by Vogelstein et al (11), has been implicated in $85 \%$ of sporadic CRC and involves the stepwise accumulation of somatic mutations leading to chromosomal instability (12). Cells acquire a growth or clonal advantage when these genomic defects activate oncogenes such as k-ras or c-myc, or inactivate tumour-suppressor genes, such as adenomatous polyposis coli (APC), deleted in colon cancer (DCC), and p53 $(13,14)$.

The second pathway is characterized by the presence of microsatellite instability (MSI) and is implicated in the development of $12-30 \%$ sporadic CRC and all cases of hereditary non-polyposis colorectal cancer (HNPCC) (15). DNA microsatellites are regions of highly polymorphic repeat nucleotide sequences mostly located in non-coding regions. The human genome contains $>100,000$ microsatellites, and genes containing microsatellites are unstable and vulnerable to mutation. CRC with high-level MSI (MSI-H) is defined by changes in the length of specific oligonucleotide sequences (16). The underlying cause of MSI is attributed to defects in DNA mismatch repair (MMR) genes. These defects occur through two pathways; inherited germline mutations such as those associated with HNPCC, or acquired somatic 
mutations. Sporadic MSI-H tumours occur through MMR gene inactivation by hypermethylation that results in silencing of hMLH1 expression. Silencing of other MMR genes (including hMSH2) implies the presence of germline mutations (17).

The phenotype of MSI-H tumours differs clinically from those demonstrating chromosomal instability. They are often more poorly differentiated and are more commonly located in the proximal colon. Despite relative resistance to fluorouracil (5FU)-based chemotherapeutic regimes, studies have also indicated an improved prognosis in patients with MSI-H tumours compared with those in stage-matched cases with chromosomal instability $(15,18,19)$. In addition, MSI-H tumours have been demonstrated to present earlier and have a lower incidence of metastases than non-MSI-H tumours (20).

While primary CRC tumours have been extensively examined, little attention has been paid to the MSI characteristics of the metastatic disease. Of the few studies that have examined the MSI status of colorectal liver metastases (CRLM), the incidence of MSI-H CRLM has been variably reported, from 3\% up to $43 \%$ (21-23). With regard to clinical outcome correlations, only one study has demonstrated an association between MSI in CRLM and improved survival, to the best of our knowledge (22).

Therefore, the current study undertook investigation of the expression of MLH1 and MSH2 proteins in resected CRLM and examined the association with clinicopathological outcome. It was hypothesized that evidence of MSI (ie. the absence of MLH1 and MSH2 expression) conveys a positive prognosis.

\section{Materials and methods}

Study cohort. Consent was obtained from patients for access to tissue for research purposes and a low-risk ethical approval was granted by the North Sydney Central Coast Area Health Human Research Ethics Committee (protocol: 0805-067M). Fifty-one consecutive patients undergoing liver resection for CRLM at the Royal North Shore Hospital campus (Sydney, Australia) between 1998 and 2006 were identified from a prospectively maintained database. Retrospective review facilitated collation of data regarding the following factors: age; gender; TNM stage; and the site of the primary CRC; use of any adjuvant chemotherapy; details of CRLM (synchronous or metachronous, number); and the development of hepatic and extrahepatic recurrence. Overall survival was measured from the date of surgery for hepatic metastases until patient mortality, or the most recent follow-up. Disease-free survival was defined as the time period from the date of liver surgery to the time of tumour recurrence. Tumour recurrence was defined as a combination of any two of the following situations: Clinical suspicion, radiological suspicion or histopathological confirmation.

Immunohistochemistry. Immunohistochemistry for MLH1 and MSH2 was performed on formalin-fixed paraffin-embedded tissue prepared from the CRLM surgical resection specimens. MLH1 antibody (cat \#550838; BD Pharmingen, BD Biosciences, Franklin Lakes, NJ, USA) and MSH2 antibody (cat \#NA27; Merck-Millipore, Darmstadt, Germany) were used at concentrations of 1:40 and 1:30, respectively. Following sectioning and heat-induced epitope retrieval, sections were incubated with the specific antibodies for $1 \mathrm{~h}$ at $25^{\circ} \mathrm{C}$. After washing, slides were incubated with the
Table I. Clinicopathological and outcome data for all cases.

\begin{tabular}{lc}
\hline Variable & $\mathrm{n}(\%$ of total $)$ \\
\hline Total cases & 51 \\
Age & median, $65 ;$ range, $29-80$ \\
Gender & \\
Male & $30(59)$ \\
Female & $21(41)$ \\
Primary tumour site & \\
Rectum & $14(27)$ \\
Left colon & $28(55)$ \\
Transverse colon & $4(8)$ \\
Right colon & $5(10)$ \\
Primary tumour stage & $24(47)$ \\
Node -ve (Dukes' A/B) & \\
Liver metastasis & $21(41)$ \\
Synchronous & $30(59)$ \\
Metachronous & mean, $2 ;$ range, $1-6$ \\
Liver metastases & mean, $42 ;$ range, $7-120$ \\
Size (mm) & \\
Post liver resection & median, $40 ;$ range, $4-92$ \\
Average follow-up time & $30(59)$ \\
Recurrence & median, $16 ;$ range, $4-37$ \\
Time to recurrence & $21(41)$ \\
Mortality & median, 32.9 \\
Disease-free survival time & median, 42.1 \\
Overall survival time & \\
\hline
\end{tabular}

Time periods, months.

MACH 4 Rabbit HRP Polymer Detection system (M4U534G; Biocare Medical, Walnut Creek, CA, USA). All slides were examined by a blinded pathologist and scored as either negative (complete absence of staining) or positive (diffuse nuclear staining). All samples demonstrated a valid internal positive control (lymphocytes, for example).

\section{Results}

Clinicopathologic variables of interest. Fifty-one patients were included in the present study ( 21 females and 30 males) and the median age was 65 years (range: 29-80) (Table I). The sites of the primary tumours were as follows: Rectum, 14 patients; left colon, 28; transverse colon, 4; and ascending colon, 5. Twenty-four patients (47\%) had node-negative primary tumours (Dukes' A and B).

Twenty-one patients (41\%) presented synchronous primary and metastatic disease (none underwent synchronous resection) and $30(59 \%)$ presented with metachronous disease. The mean number of metastases was 2 (range: 1-6), with 28 patients (54\%) having solitary metastasis. Bilobar disease was present in 14 patients $(27 \%)$ and the mean CRLM tumour size was $42 \mathrm{~mm}$ (range: $7-120 \mathrm{~mm}$ ).

Seventeen patients $(33 \%)$ underwent neoadjuvant 5FU-based chemotherapy, and five had adjuvant chemotherapy. 
Two patients with rectal cancer had undergone neoadjuvant chemoradiotherapy.

During the average post-liver resection follow-up interval of 40 months (range: 4-92) there were thirty recurrences. Eighteen were intrahepatic and twelve were extrahepatic (lung, 3; peritoneal, 3; brain, 2; pelvic, 2; adrenal, 1; and kidney, 1). The median time to recurrence was 16 months (range: 4-37 months). Of the thirty patients with disease recurrence, six underwent further surgery (hepatic, 4; pulmonary, 1; and cerebral, 1). The median overall survival from the time of liver resection was 42.1 months and disease-free survival was 32.9 months. There were a total of 21 disease-related mortalities during the follow-up period.

Immunohistochemistry. All of the samples demonstrated diffuse positive nuclear staining for MLH1 and MSH2. No samples displayed the staining pattern characteristic of MSI-H tumour cells (i.e. loss of MMR staining). Given the absence of MMR deficiency in this cohort, it was not possible to identify statistical correlations between this factor and clinical outcome.

\section{Discussion}

The investigation of tumour MSI has become routine in colorectal practice following the validation by the National Cancer Institute of a panel of five microsatellites (16). These have allowed standards for classifying tumours into MSI-high (H), MSI-low (L), or microsatellite-stable (MSS) groups (24). Immunohistochemical techniques are now widely available to assess the level of gene product expression in standard formalin-fixed, paraffin-embedded sections and this has become the favoured technique for the investigation of MSI status in colorectal cancer $(16,18)$. Nuclear staining for MMR genes is ubiquitous throughout human tissue, even in cancer. Positive staining demonstrates the absence of MMR and MSI. Conversely, loss of expression denotes the presence of MSI specific to the MMR protein being that is stained for.

The incidence of MSI in primary CRC is $\sim 15-20 \%$. These tumours display distinctive clinicopathological features, including a tendency to be located in the right colon, associations with other neoplasms, a younger age at presentation, a high grade or medullary type, mucinous differentiation, and peritumoural ('Crohn's like') lymphoid infiltration. MSI status is also associated with an earlier stage at presentation and less nodal involvement compared with MSS tumours (25).

Overall, the presence of MSI in primary sporadic CRC has been repeatedly demonstrated to be an independent predictor of improved overall and disease-free survival (15,26,27). A number of explanations have been suggested for this finding: Genes with MSI have been demonstrated to mutate at rates up to 100-fold higher than MSS cells. Thus, the rate of gene defects may be higher and such defects may either be lethal to the cell or result in the production of dysfunctional cell-cycle proteins that act as a brake on tumour progression (28). Secondly, MSI cancers demonstrate increased peritumoural lymphoid infiltration and contain high numbers of activated cytotoxic lymphocytes (23). This may be due to defective MMR, which leads to the production of antigenic proteins that stimulate a cytotoxic immune response within the tumour.

In the current study, none of the 51 patients exhibited an absence of MMR gene product staining consistent with MSI-H metastases. These results are similar to those of a number of previous studies. For example, Thorstensen et al (8) presented a liver metastasis MSI rate of only $2.7 \%$. This contrasts with the results of Messick et al (9) who reported a rate of $10 \%$. The authors explained their increased incidence by the inclusion of rectal cancers that demonstrated a very low MSI rate, and the use of a genetic panel for the diagnosis of MSI, which potentially included tumours that other studies would not have classified as MSI-H. In the present study, $27 \%$ of samples were rectal CRLM. In general, cases of CRLM appear to maintain relatively low MSI rates when compared with corresponding primary tumours, which consistently maintain MSI rates of 15-20\%. This difference has been indicated to be statistically significant in at least one matched study (10). The absence of data regarding the MSI status of primary tumours in the present study precludes a discussion regarding corresponding expression.

There are a number of potential explanations for the discrepancy in MSI rates between primary and secondary cancers. MSI-H tumours may not metastasise to the liver as readily as MSI-L or MSS tumours. Furthermore, they may metastasise in a different pattern. The present surgical cohort also represents a select population, in terms of patients (fit for surgery) and disease (isolated to the liver and surgically resectable). MSI-H tumours, having developed the potential to metastasise, may spread to the liver in such an aggressive fashion that they are surgically unresectable, or progress so rapidly as to render patients unfit for potential liver resection. However, to establish this hypothesis, the routine use of biopsy and determination of MSI status of all CRLM is required; a practice that would not be clinically advisable. Additionally, numerous patients with CRLM have often received chemotherapy as adjuvant or increasingly neoadjuvant treatment prior to hepatectomy. The paucity of MSI-H cells in liver metastases may reflect a greater sensitivity of these tumours to various chemotherapeutic regimens; a fact that has been noted previously (29). Another possibility is that MSI-H primary tumours harbour a subpopulation of MSS cells that possess greater metastatic potential and are overly represented by clonal selection in liver metastases (10). Whilst plausible, data from a range of types of primary cancer (including CRC) and corresponding metastases suggest that liver metastases exhibit MSI at the same loci as the primary colonic tumour (30,31).

The use of translational molecular phenotyping for the management of CRC is increasing. Measurement of MSI status and other mutations such as KRAS and BRAF are now being used to guide tailored treatment. More specifically, studies investigating the response of MSI-H primary tumours to flouropyrimidine-based chemotherapy have produced conflicting results $(19,29,30,32,33)$. However, the weight of evidence now strongly suggests a significantly reduced benefit for MSI-H compared with MSS tumours with nodal involvement treated with 5FU-based regimes $(15,18)$. Studies demonstrate no significant difference between the response of MSI-H and MSS tumours that present metastasis to combinatorial chemotherapy regimens, as concluded in a review by Des Guetz et al (34).

The surgical resection of metastases is a well-established treatment for not only liver, but lung and cerebral metastases from CRC. The aggressive treatment of what was previously thought to be terminal disease has necessitated the identification of prognostic markers of success for these potentially morbid 
resections. While the indicators for hepatectomy are expanding, clinical risk factors for intra- and extra-hepatic recurrence have been reported. Molecular markers are increasingly being sought for the chemotherapeutic and surgical treatment of metastatic disease, in addition to those related to the primary tumour. It is tempting to extrapolate molecular knowledge regarding primary CRC to the metastatic setting; however, as in the MMR status of primary CRC and corresponding CRLM, there may exist significant differences. This is not only true for MLH1 and MSH2 mutations, but has also been reported for other markers such as thymidylate synthase and Bax (35).

As demonstrated in the present study, the low frequency of MMR deficiency in liver metastases precludes its use as either a predictor of outcome, or as a selection criterion for liver surgery and/or adjuvant therapy. This highlights the requirement for ongoing research to identify molecular markers of translational significance for management of colorectal liver metastases.

\section{References}

1. Jemal A, Bray F, Center MM, Ferlay J, Ward E and Forman D: Global cancer statistics. CA Cancer J Clin 61: 69-90, 2011.

2. Wolpin BM and Mayer RJ: Systemic treatment of colorectal cancer. Gastroenterology 134: 1296-1310, 2008.

3. Cunningham JM, Kim CY, Christensen ER, Tester DJ, Parc Y, Burgart LJ, Halling KC, McDonnell SK, Schaid DJ, Walsh Vockley $\mathrm{C}$, et al: The frequency of hereditary defective mismatch repair in a prospective series of unselected colorectal carcinomas. Am J Hum Genet 69: 780-790, 2001.

4. Aaltonen LA, Peltomäki P, Leach FS, Sistonen P, Pylkkänen L, Mecklin JP, Järvinen H, Powell SM, Jen J, Hamilton SR, et al: Clues to the pathogenesis of familial colorectal cancer. Science 260: 812-816, 1993.

5. Fong Y, Fortner J, Sun RL, Brennan MF and Blumgart LH: Clinical score for predicting recurrence after hepatic resection for metastatic colorectal cancer: analysis of 1001 consecutive cases. Ann Surg 230: 309-321, 1999

6. Simmonds PC, Primrose JN, Colquitt JL, Garden OJ, Poston GJ and Rees M: Surgical resection of hepatic metastases from colorectal cancer: a systematic review of published studies. Br J Cancer 94: 982-999, 2006.

7. Pawlik TM and Choti MA: Shifting from clinical to biologic indicators of prognosis after resection of hepatic colorectal metastases. Curr Oncol Rep 9: 193-201, 2007.

8. Thorstensen L, Qvist H, Nesland JM, Giercksky KE and Lothe RA: Allelotype profiles of local recurrences and distant metastases from colorectal-cancer patients. Int J Cancer 69: 452-456, 1996

9. Messick CA, Church J, Casey G and Kalady MF: Identification of the methylator (serrated) colorectal cancer phenotype through precursor serrated polyps. Dis Colon Rectum 52: 1535-1541, 2009.

10. Salahshor S, Kressner U, Fischer H, Lindmark G, Glimelius B, Pâhlman L and Lindblom A: Microsatellite instability in sporadic colorectal cancer is not an independent prognostic factor. Br J Cancer 81: 190-193, 1999.

11. Vogelstein B, Fearon ER, Hamilton SR, Kern SE, Preisinger AC, Leppert M, Nakamura Y, White R, Smits AM and Bos JL: Genetic alterations during colorectal-tumor development. N Engl J Med 319: 525-532, 1988.

12. Fearon ER and Vogelstein B: A genetic model for colorectal tumorigenesis. Cell 61: 759-767, 1990.

13. Leslie A, Carey FA, Pratt NR and Steele RJ: The colorectal adenoma-carcinoma sequence. Br J Surg 89: 845-860, 2002.

14. Boland CR, Thibodeau SN, Hamilton SR, Sidransky D, Eshleman JR, Burt RW, Meltzer SJ, Rodriguez-Bigas MA, Fodde R, Ranzani GN and Srivastava S: A National Cancer Institute Workshop on Microsatellite Instability for cancer detection and familial predisposition: development of international criteria for the determination of microsatellite instability in colorectal cancer. Cancer Res 58: 5248-5257, 1998.

15. Walther A, Johnstone E, Swanton C, Midgley R, Tomlinson I and Kerr D: Genetic prognostic and predictive markers in colorectal cancer. Nat Rev Cancer 9: 489-499, 2009.
16. Poynter JN, Siegmund KD, Weisenberger DJ et al; Colon Cancer Family Registry Investigators: Molecular characterisation of MSI-H colorectal cancer by MLH1 promoter methylation, immunohistochemistry and missmatch repair germline mutation screening. Cancer Epidemiol Biomarkers Prev 17: 3208-3215, 2008.

17. Popat S, Hubner R and Houlston RS: Systematic review of microsatellite instability and colorectal cancer prognosis. J Clin Oncol 23: 609-618, 2005

18. Jover R, Payá A, Alenda C, Poveda MJ, Peiró G, Aranda FI and Pérez-Mateo M: Defective mismatch-repair colorectal cancer: clinicopathologic characteristics and usefulness of immunohistochemical analysis for diagnosis. Am J Clin Pathol 122: 389-394, 2004.

19. Jover R, Zapater P, Castells A et al; Gastrointestinal Oncology Group of the Spanish Gastroenterological Association: The efficacy of adjuvant chemotherapy with 5-flourouracil in colorectal cancer depends on mismatch repair status. Eur J Cancer 45: 365-373, 2009.

20. Malesci A, Laghi L, Bianchi P et al: Reduced likelihood of metastases in patients with microsatellite-unstable colorectal cancer. Clin Cancer Res 13: 3831-3839, 2007.

21. Haddad R, Ogilvie RT, Croitoru M, Muniz V, Gryfe R, Pollet A, Shanmugathasan P, Fitzgerald T, Law $\mathrm{CH}$, Hanna SS, et al: Microsatellite instability as a prognostic factor in resected colorectal cancer liver metastases. Ann Surg Oncol 11: 977-982, 2004.

22. Chen WS, Chen JY, Liu JM, Lin WC, King KL, Whang-Peng J and Yang WK: Microsatellite instability in sporadic-colon-cancer patients with and without liver metastases. Int J Cancer 74: 470-474, 1997.

23. Ishimaru G, Adachi J, Shiseki M, Yamaguchi N, Muto T and Yokota J: Microsatellite instability in primary and metastatic colorectal cancers. Int J Cancer 64: 153-157, 1995.

24. Nicholl ID and Dunlop MG: Molecular markers of prognosis in colorectal cancer. J Natl Cancer Inst 91: 1267-1269, 1999.

25. Ding SF, Delhanty JD, Zografos G, Michail NE, Dooley JS and Habib NA: Chromosome allele loss in colorectal liver metastases and its association with clinical features. Br J Surg 81: 875-878, 1994.

26. Samowitz WS and Slattery ML: Regional reproducibility of microsatellite instability in sporadic colorectal cancer. Genes Chromosomes Cancer 26: 106-114, 1999.

27. Moslein G, Tester DJ, Lindor NM, Honchel R, Cunningham JM, French AJ, Halling KC, Schwab M, Goretzki P and Thibodeau SN: Microsatellite instability and mutation analysis of hMSH2 and hMLH1 in patients with sporadic, familial and hereditary colorectal cancer. Hum Mol Genet 5: 1245-1252, 1996.

28. Kochhar R, Halling KC, McDonnell S, Schaid DJ, French AJ, O'Connell MJ, Nagorney DM and Thibodeau SN: Allelic imbalance and microsatellite instability in resected Duke's D colorectal cancer. Diagn Mol Pathol 6: 78-84, 1997.

29. Bendardaf R, Lamlum H, Ristamäki R, Korkeila E, Syrjänen K and Pyrhönen S: Mismatch repair status is a predictive factor of tumour response to 5-fluorouracil and irinotecan chemotherapy in patients with advanced colorectal cancer. Tumour Biol 28: 212-220, 2007.

30. Feeley KM, Fullard JF, Heneghan MA, Smith T, Maher M, Murphy RP and O'Gorman TA: Microsatellite instability in sporadic colorectal carcinoma is not an indicator of prognosis. J Pathol 188: 14-17, 1999.

31. Halling KC, French AJ, McDonnell SK, Burgart LJ, Schaid DJ, Peterson BJ, Moon-Tasson L, Mahoney MR, Sargent DJ, O'Connell MJ, et al: Microsatellite instability and $8 \mathrm{p}$ allelic imbalance in stage B2 and C colorectal cancers. J Natl Cancer Inst 91: 1295-1303, 1999.

32. Lothe RA, Peltomäki P, Meling GI, Aaltonen LA, Nyström-Lahti M, Pylkkänen L, Heimdal K, Andersen TI, Møller P, Rognum TO, et al: Genomic instability in colorectal cancer: relationship to clinicopathological variables and family history. Cancer Res 53: 5849-5852, 1993.

33. Elsaleh H and Iacopetta B: Microsatellite instability is a predictive marker for survival benefit from adjuvant chemotherapy in a population-based series of stage III colorectal carcinoma. Clin Colorectal Cancer 1: 104-109, 2001

34. Des Guetz G, Uzzan B, Nicolas P, Schischmanoff O, Perret GY and Morere JF: Microsatellite instability does not predict the efficacy of chemotherapy in metastatic colorectal cancer. A systematic review and meta-analysis. Anticancer Res 29: 1615-1620, 2009

35. Huang J, Kuismanen SA, Liu T, Chadwick RB, Johnson CK, Stevens MW, Richards SK, Meek JE, Gao X, Wright FA, et al: MSH6 and MSH3 are rarely involved in genetic predisposition to nonpolypotic colon cancer. Cancer Res 61: 1619-1623, 2001. 